\title{
Correlative Fluorescence and Liquid Cell STEM of Live Magnetotactic Bacteria
}

Taylor Woehl, ${ }^{1}$ Sanjay Kashap, ${ }^{1}$ Miguel Sánchez-Quesada, ${ }^{2}$ Concepción Jiménez López, ${ }^{2}$ Teresa

Perez-Gonzalez, ${ }^{3}$ Damien Faivre, ${ }^{3}$ Dennis Trubytsyn, ${ }^{4}$ Dennis Bazylinski, ${ }^{4}$ Tanya Prozorov ${ }^{1}$

${ }^{1}$ Division of Materials Science and Engineering, Ames DOE Laboratory, Ames, IA 50011, USA
${ }^{2}$ Department of Microbiology, University of Granada, Spain
${ }^{3}$ Department of Biomaterials, Max Plank Institute for Colloids and Interfaces, Science
Park Golm, 14424 Potsdam, Germany
${ }^{4}$ Department of Biological Sciences, University of Nevada-Las Vegas, Las Vegas, NV 89154, USA

Magnetotactic bacteria, present in many natural aquatic environments, biomineralize ordered chains of uniform magnetite or greigite nanocrystals, also known as magnetosomes. These nanoparticles exhibit nearly perfect crystal structures, faceting, and consistent species-specific morphologies, leading to well-defined magnetic properties. As a result, magnetotactic bacteria can serve as a model system for the study of the molecular mechanisms for magnetite biomineralization. Transmission electron microscopy (TEM) can provide critical information about the organization of the magnetosomes, and provide critical information about the growth mechanisms by revealing the nanoparticle structure on the atomic level. Conventional TEM traditionally does not allow imaging in native liquid or atmospheric environments because of the high vacuum of the specimen enclosure. Even with advanced cryo-TEM imaging, it is inherently difficult to distinguish the individual components in bacterial specimens due to notoriously low contrast of a biological matter. Therefore direct observation of the time-dependent growth of magnetosomes is a significant challenge.

Liquid cell TEM is a powerful technique for observing dynamic processes in liquid on the nanoscale, with applications ranging from nanoparticle growth [1,2] to cellular imaging[3]. Liquid cell STEM has been used to image eukaryotic cells, where high contrast materials such as gold nanoparticles [4] were employed to label individual proteins in the cells. Correlative fluorescence microscopy and liquid cell STEM have allowed researchers to correlate the ultrastructure of cells with microscale cellular functions $[5]$.

Here we use correlative fluorescence and liquid cell scanning TEM (STEM) to image live magnetotactic bacteria. We employed a continuous flow fluid cell TEM platform produced by Hummingbird Scientific to image AMB-1 magnetotactic bacteria in liquid. The bacteria were imaged using a high angle annular dark field (HAADF) detector, where the high atomic number magnetosomes chains in the bacteria provided high contrast allowing imaging of the bacteria. Bacteria were observed to move under the electron beam, suggesting that they are alive in the liquid cell. To confirm the bacteria's viability, a fluorescent dye mixture was flowed through the fluid cell-bacteria that were alive were stained with a green fluorescent protein, while dead cells were stained red with propidium iodide. This technique should be generally applicable for correlative imaging of a broad range of gram-negative bacteria. Correlative imaging of live bacteria is a first step in directly observing biomineralization of magnetosomes in live magnetotactic bacteria [6]. 


\section{References:}

[1] H. M. Zheng, et al. Science 324 (2009), p. 1309.

[2] T. J. Woehl, et al. Acs Nano 6, p. 8599.

[3] N. de Jonge, et al. Proceedings of the National Academy of Sciences of the United States of America 106 (2009), p. 2159.

[4] D. B. Peckys and N. de Jonge, Nano Letters 11, 1733 (2011).

[5] M. J. Dukes, et al. Acs Nano 4, 4110 (2010).

[6] The work in Ames Laboratory was supported by the U.S. Department of Energy, Basic Energy Sciences, Materials Science and Engineering Division. The Ames Laboratory is operated for the U.S. Department of Energy by Iowa State University under contrat DE-AC02$07 \mathrm{CH} 11358$.
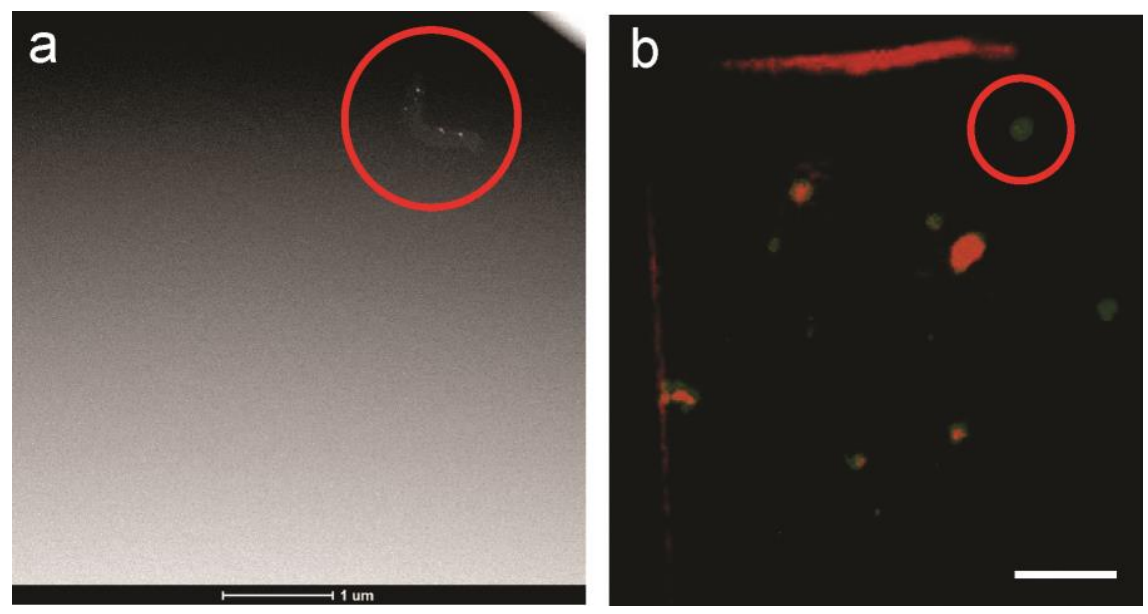

Figure 1. Correlative liquid cell STEM and fluorescence imaging of AMB-1 bacteria. (a) HAADF STEM image of AMB-1 bacteria. The bright spots in the bacteria are magnetosomes. (b) Fluorescence image of the same sample as (a). Green and red fluorescent channel images are overlaid to show live (green) and dead (red and green) bacteria. The bacteria circled in red is the same as in (a). Scale bar is $10 \mu \mathrm{m}$. 